\title{
Euclid: an optical-NIR all sky space mission for Cosmology
}

\author{
R. Scaramella ${ }^{1}$ and EIC \& ENIS consortia
}

\author{
INAF - Osservatorio Astronomico di Roma
}

\begin{abstract}
We give an overview of Euclid, a space mission selected for study within ESA's Cosmic Vision framework. Euclid is a survey mission which aims to deeply cover the whole extragalactic sky $(2 \pi$ sr). Exquisite optical imaging in one wide optical band plus deep photometry in three NIR bands will allow to map gravitational lensing across the sky, while NIR spectroscopy will allow a full sky sample of accurate galaxy redshifts up to $z \sim 2$. These measures will allow to probe with unprecedented accuracy presence and characteristics of Dark Energy, Dark Matter and possible presence of non standard gravity. The giant database will also have an enormous legacy value and allow the exploitation of countless science topics
\end{abstract}

\section{Introduction}

The current Euclid mission concept stems from two dark energy related missions which were proposed to ESAs Cosmic Vision programme and were jointly selected: the Dark UNiverse Explorer (DUNE) and the SPectroscopic All-sky Cosmology Explorer (SPACE). Initial studies in 2008 by an ESA ad hoc advisory panel led to the Euclid merged concept, an ESA mission comprising two instruments: a two channels optical \& NIR imager, and a NIR spectrograph. These instruments were proposed, are studied and will provided by the Euclid Imaging Consortium [EIC] (A. Réfregiér P.I.) and the Euclid Near Infrared Spectrograph Consortium [ENIS] (A. Cimatti P.I.), respectively. These consortia are formed by hundreds of scientists from several countries. Euclid has been the object of an Assessment Phase in 2008-2009 in the context of ESAs Cosmic Vision programme and is a candidate for selecrion for the first ESA's medium mission launch slot in late 2017.

Euclid primary goal is to map the geometry and evolution of the dark universe with unprecedented precision. The aim is to place high accuracy constraints on Dark Energy, Dark Matter and Gravity using two independent cosmological probes: weak gravitational lensing (WL) and baryonic acoustic oscillations (BAO). For this purpose, Euclid will measure the shape and spectra of galaxies over the entire extragalactic sky in the visible and NIR, out to redshift 2, thus covering the period over which dark energy accelerated the universe expansion ( $<10$ Billion years). Supernovæ, galaxy clusters and the Integrated Sachs-Wolf effect will be used as secondary cosmological probes. The Euclid datasets will also provide a unique legacy surveys for the study of galaxy evolution, large-scale structure, the search for high redshift objects and for various other fields of astronomy.

The baseline mission concept (Laureijs et al. 2009) is based on a telescope with a primary mirror of $1.2 \mathrm{~m}$ diameter. The payload baseline comprises wide field instruments $\left(0.5 \mathrm{deg}^{2}\right)$ : an imaging instrument comprising a visible and a NIR channel, and a NIR spectroscopic instrument. The visible channel is used to measure the shapes of galaxies for weak lensing, with a resolution of 0.18 arcsec in a wide visible red band $(\mathrm{R}+\mathrm{I}+\mathrm{Z}$,
$0.55-0.92 \mu \mathrm{m})$. The NIR photometric channel provides three NIR bands (Y, J, $\mathrm{H}_{w}$, spanning 1.0-2.0 $\mu \mathrm{m}$ ) with a resolution of 0.3 arcsec. The baseline for the NIR spectroscopic channel operates in the wavelength range $1.0-2.0 \mu \mathrm{m}$ in slitless mode at a spectral resolution $\mathrm{R} \sim 500$, employing 0.5 " pixels. An optional spectroscopic implementation is slit spectroscopy using digital micro-mirror devices (DMDs).

The mission will perform a wide survey of the entire ex $\Omega$ tragalactic sky $\left(20,000 \mathrm{deg}^{2}\right)$ down to $24.5 \mathrm{AB}$ magnitude in the visible, thus providing 30-40 resolved galaxies per $\mathrm{amin}^{2}$ For all galaxies, photometric redshifts are obtained from the broad-band visible and near-IR measurements and complementary ground-based observations in other visible bands. For 4060 million galaxies with $\mathrm{H} \alpha$ line flux level $>4 \cdot 10^{-16} \mathrm{erg} \mathrm{s}^{-1}$ $\mathrm{cm}^{-2}$ the slitless spectrometer will directly measure the redshift with a success rate in excess of $35 \%$.

A two magnitude deeper survey of $\sim 40$ square degrees will also be performed to monitor the stability of the spacecraft and payload and for legacy science.

The factors that require a space missions are the need for a small and stable optical spread function and the reduced back $f$ ground for deep NIR photometry and spectroscopy .

Planned characteristics and science topics of the mission are given in detail in the Assessment Study ("Yellow Book", Laureijs et al. 2009, hereafter EYB). Further details, additional topics, related bibliography and additional references can be found in the EYB, in the EIC Science Book (hereafter EICSB, Réfrégier et al. 2009), and in the proceedings of the meeting "Observing the Dark Universe with Euclid" (ESTEC, Noordwijk, Nov 2009, http://www . congrex.nl/09c08)

\section{Theory and quest for observable, discriminating parameters}

In the last decade from new measurements of Supernovæ and of the anisotropies in the cosmic microwave background (CMB), a coherent picture started to emerge: even though the Universe appears to be spatially flat, matter only makes up $25 \%$ of the critical energy today, and the rest is something else, for which the best-known candidate is the cosmological constant $\Lambda$. But it 
is not only the $75 \%$ dark energy that is puzzling. Both big-bang nucleosynthesis (BBN) and the CMB indicate that baryons (the "normal" matter) only make up about $4 \%$ of the energy density today. The remaining $21 \%$ are an unknown substance called dark matter because it is apparently invisible but clusters at least on large scales like pressureless matter.

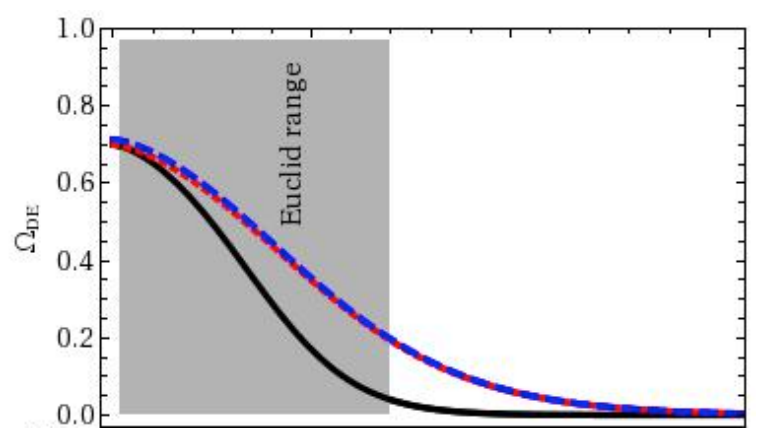

Fig. 1: Effect of dark energy on the evolution of the Universe. Fraction of the density of the Universe in the form of dark energy as a function of redshift $\mathrm{z}$., for a model with a cosmological constant $(\mathrm{w}=-1$, black solid line), dark energy with a different equation of state $(w=-0.7$, red dotted line), and a modified gravity model (blue dashed line). In all cases, dark energy becomes dominant in the low redshift Universe era probed by Euclid, while the early Universe is probed by the CMB.

In general, given the plethora of possible theoretical models, it is useful to adopt a phenomenological approach and therefore some parametrisations. In the dark energy context tne main parameter is the one for equation of state of the dark energy component, $w \equiv p / \rho$. If we can consider the Universe as evolving like a homogeneous and isotropic FriedmannLemaître-Robertson-Walker (FLRW) universe, then the only observationally accessible quantity is the expansion rate of the universe $H$, given by the Friedmann equation, $H(a)^{2}=$ $(\dot{a} / a)^{2}=(8 \pi G / 3)\left[\rho_{m}(a)+\rho_{\mathrm{DE}}(a)\right]$. This equation governs the expansion law of the Universe as whole and can be studied with geometrical tests: luminosity and angular diameter distances are determined by integrals of $1 / H$, and $H$ itself can be directly measured by a number of methods.

The dark energy is described by its homogeneous energy density $\rho_{\mathrm{DE}}$ and the isotropic pressure $p_{\mathrm{DE}}$, diagonal elements of the energy momentum tensor. Any other non-zero component of the latter would require us to go beyond the FLRW description of the Universe. The evolution of $\rho$ is then governed by the covariant conservation equations which in this case reduce simply to

$$
\dot{\rho}_{\mathrm{DE}}=-3 H\left(\rho_{\mathrm{DE}}+p_{\mathrm{DE}}\right)=-3 H(1+w) \rho_{\mathrm{DE}} .
$$

Conclusions can be drawn from the phenomenological $w(a)$ : if the observed $w$ ever deviates significantly from -1 then a cosmological constant is ruled out, and if $w<-1$ then canonical scalar field models of the dark energy are in trouble. Once $H(a)$ has been measured with the needed accuracy, then $w(a)$ can be extracted. For an evolving $w(a)$ a number of models can be described by the parametrization (Chevallier \& Polarski 2001, Linder 2003) $w(a)=w_{p}+\left(a_{p}-a\right) w_{a}$ obtained by Taylor expansion around a pivot expansion factor, $a_{p}$, which renders errors on $w_{p}$ and $w_{a}$ uncorrelated (often one normalizes at present where $a=1$ and the parameters plane is $w_{0}-w_{a}$ ). Then the ability of a given experiment to measure the DE equation of state can be expressed (Albrecht et al. 2005) in terms of a "Figure-of-Merit" [FoM], given by FoM $=1 /\left(\Delta w_{p} \times \Delta w_{a}\right)$.

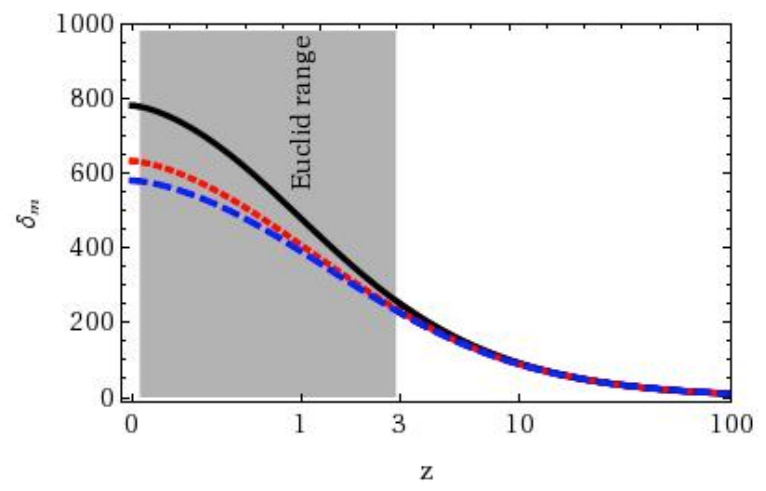

Fig. 2: Growth factor of cosmic structures for the same three models in Fig. 1. Only by measuring the geometry and the growth of structure at low redshifts can a modification of dark energy be distinguished from that of gravity.

The latter $\Delta$ are obtained by marginaliziation in the Fisher Matrix over the many other typical parameters of the models, such as f.i. details of the power spectrum (amplitude, $\sigma_{8}$, primordial spectral index, $n$ ). The discriminating power of a given experiment then can immediately be expressed graphically by ellipses in the $w_{a}-w_{p}$ plane and confronted with models predictions in the same plane (in the $w_{0}-w_{a}$ plane the ellipses are tilted, since the parameters are correlated). The FoM is inversely proportional to the ellipse area. By combining present experiments this is $\sim O(10)$ (Komatsu et al. 2009), while Euclid will yield $\sim 500$ by itself and will reach $\sim 1500$ by adding the information which will be provided by Planck (EYB).

However, there is an ambiguity present since it is possible, for instance, to ascribe the expansion history to a scalar field potential or equivalently to construct a function $f$ so that a $f(R)$ type (in the Lagrangian) modified gravity model yields the same expansion history. Either explanation is indistinguishable at the background level and therefore one needs more information, different from $H(a)$, and so to look study additional quantities, such as density perturbations.

Therefore the growth-rate of the matter perturbations comes into play. In the standard picture, once $H(a)$ is accurately known, the dark matter perturbations evolve according to $\ddot{\delta}_{m}+2 H \dot{\delta}_{m}=4 \pi G \rho_{m} \delta_{m}$. A common parametrisation is in terms of the parameter $\gamma$, the matter growth index (Wang \& Steinhardt 1998)

$$
\frac{d \log \delta_{m}}{d \log a} \equiv f(a) \cong \Omega_{m}(a)^{\gamma} .
$$

In this simple case, $\gamma$ is uniquely fixed by the expansion history, which in turn depends on $w(a)$. A good fit to the full numerical result is $\gamma \approx 0.55+0.05[1+w(a=0.5)]$ (Linder 2005). The 
above description, which has only one parameter, assumes both Poisson equation and that only Dark Matter contributes to the density perturbation source term, i.e. $\Delta \phi=4 \pi G \rho \delta_{m}$.

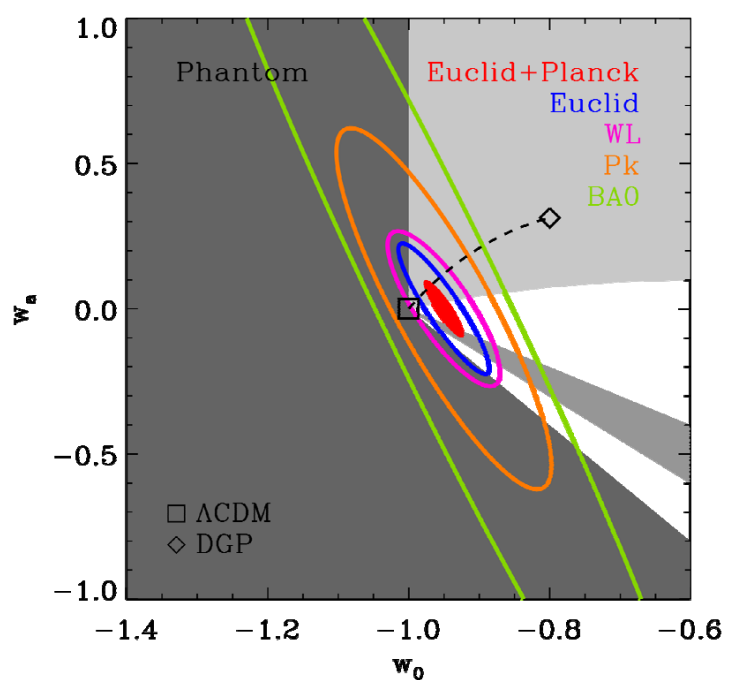

Fig. 3: Predicted constraints from Euclid on the dark energy w0-wa plane for a $w_{0}=-0.95$ reference model. The outer (green) ellipses show the constraints from $\mathrm{BAO}$, orange shows the galaxy power spectrum, $\mathrm{P}(\mathrm{k})$, purple weak lensing alone, and inner blue ellipse the combined Euclid probes. The inner red ellipse is the combined Eulcid and Planck constraints. The square denotes $\Lambda \mathrm{CDM}$ and diamond DGP in parameter space, with the dotted line connecting them showing where extended DGP models lie.

Now, in order to consider a wider class of possible models one then needs to study perturbations at a more general level by the considering the metric element

$$
d s^{2}=a(\tau)^{2}\left[-(1+2 \psi) d \tau^{2}+(1-2 \phi) d r^{2}\right]
$$

where the two functions of position and time $\psi$ and $\phi$ play a role very similar to gravitational potentials. It is worth noticing that massive particles will be influenced practically only by $\psi$, while massless ones, such as lensed photons, will feel the difference of the two (EICSB).

At the background, unperturbed level, the evolution of the universe is described by $H$, which is linked to $\rho$ by the Einstein equations, and $p$ controls the evolution of $\rho$, but a priori it is a free quantity describing the physical properties of the fluid. Therefore in addition to the standard picture now there are $\psi$ and $\phi$ describing the Universe, and they are linked to $\delta \rho$ and peculiar $v$ of the fluids through the Einstein equations. Pressure perturbations $\delta p$ and anisotropic stress $\pi$ in turn describe the fluids. This means that a general dark energy component can be described by phenomenological parameters similar to $w$, even at the level of first order perturbation theory. This description adds the two new parameters $\delta p$ and $\pi$, which are both functions of scale as well as time. These parameters fully describe the dark energy fluid, and they can in principle be measured (see Fig. 2).
An alternative to invoking the presence of dark fluids in the energy momentum tensor is to slightly modify GR itself to explain the accelerated expansion. However, these deviations can still be recast in the form $G_{\mu \nu}=-8 \pi G T_{\mu \nu}-Y_{\mu \nu}$, where $Y_{\mu \nu}$ can be intepreted again as the presence of a fluid with an effective anisotropic stress and an pressure perturbation, i.e. a specific ad hoc Dark Energy model. Therefore at the linear perturbation level both the dark energy perturbations or the modifications of gravity can be described by two additional functions. A single extra parameter, for example only $\gamma$, does not suffice.

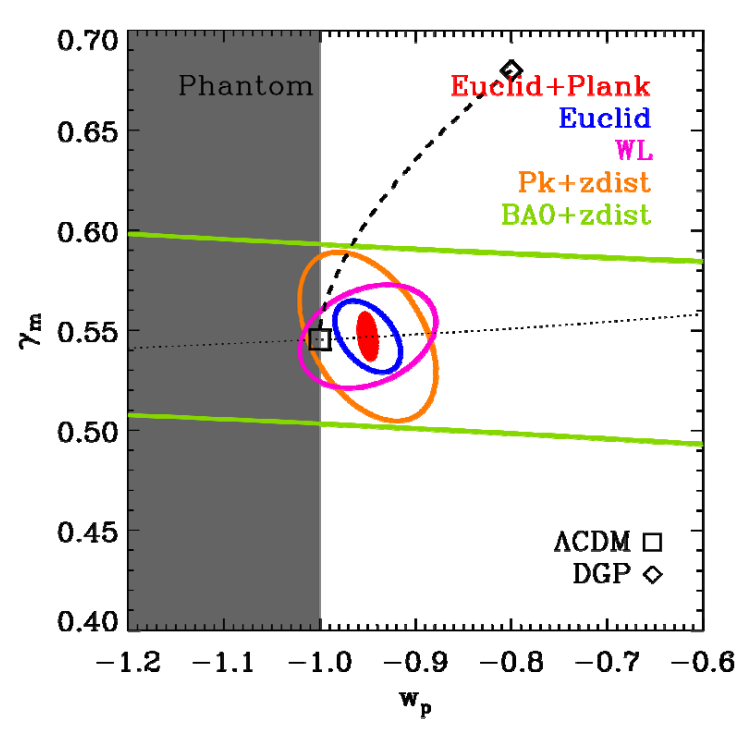

Fig. 4: Constraints similar to those in Fig. 3 but in the $w_{p}$ and $\gamma_{m}$ plane

The two potential functions $\phi(k, a)$ and $\psi(k, a)$ can in general be recast in terms of other, simpler parameters such as the dimensionless quantities $Q$ (related to the DE clustering and the modification of Poisson eq) and $\eta$ (related to the difference $\psi-\phi$ and hence to the anisotropic stress), which can more easily be related to observable quantities. For gravitational lensing it is useful to consider $\Sigma \equiv Q(1+\eta / 2)$. Since there is also a relation between $\gamma$ and $Q(1+\eta)$, in the limit of small deviations from the canonical GR values, one has (Amendola et al. 2008)

$$
\gamma=\gamma_{s t d}\left[1+\frac{Q(1+\eta)-1}{(w-1)\left(1-\Omega_{m}\right)}\right]
$$

where $\gamma_{s t d}$ is the standard value (i.e. for uncoupled, unclustered dark energy in Einstein gravity). A significant deviation of $\gamma$ from $\gamma_{s t d}$ would amount to the discovery that either dark energy clusters or that gravity is modified. Therefore to the FoM ellipses in the $w_{p}-w_{a}$ plane now add the ones in the $\gamma-w_{p}$ plane, where gravity models (such as the popular DGP, Dvali et al. 2000) can more easily be separated from DE ones (in general the formers, differently from the latters, typically have a non zero $\pi$ (EICSB). 


\section{Main Probe: Gravitational Lensing}

Image distortions from light deflection due to gravitational potential wells can range from weak (round object get very slightly elongated), to intermediate (deformations to banana shapes, flexion) or even strong (extreme deformations to elongated arcs and multiple images). The "cosmic shear" refers to the the Weak lensing (WL) regime and in particular can be used to reconstruct the large-scale distribution of mass along the line-of-sight, unlike other methods that probe the distribution of light. WL measurements can thus be directly compared with theoretical models of structure formation and are powerful tool to measure cosmological parameters (Refregier 2003, Heavens et al., 2006). WL probes both the geometry of the Universe [and thus $H(z)]$ and the amplitude of the power spectrum, $\sigma_{8}(z)$, at different redshifts, which gives directly the growth factor $G(z)$ of mass perturbations.

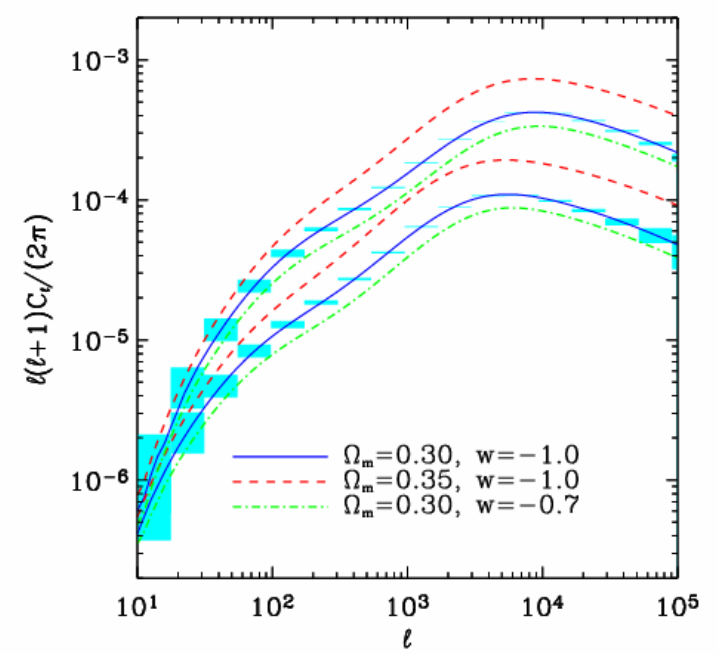

Fig. 5: Cosmic shear angular power spectrum predicted at $z=0$ and $z=0.5$ for a number of models.

To reveal with sufficient significance the extremely weak shear signal (typically the angular power spectrum, see Fig. 5; it is also possible to study cross power spectra at different $z$ ), one needs to sample large effective volumes with deep and excellent images in order to spatially resolve a large number of lensed galaxies per square arc minute. Hence the need of surveying very large areas of the sky in very good seeing and stable conditions. The latter requirements brings naturally to space observations, as demonstrated by the results obtained using HST on the COSMOS field (Massey et al. 2007).

However, this requires both high accuracies in the shear measurement and a good knowledge of the redshift distribution of the background (source) galaxies. For the latter, in order to measure the signal and the power spectrum for several different redshift bins (tomography), one needs for the lensed galaxies in a redshift bins to have small bias on the mean, $\sigma_{\langle z\rangle}<2 \cdot 10^{-3}(1+z)$, and small dispersion on individual measurements, $\sigma_{z}<5 \cdot 10^{-2}(1+z)$. These can be achieved (Bordoloi et al. 2009) by culling the samples and by complementing
Euclid photometry with that from planned ground based surveys.

Also systematic sources of uncertainty (not only instrumental ones, e.g. effects due to galaxies instrinsic alignments) should be kept at minimum: for Euclid one needs for the shear $\sigma_{s y s}^{2}<10^{-7}$ (Amara \& Réfregiér 2008). In the last years analysis methods have been constantly improving by independent cross-checks and new approaches (GREAT08, Bridle et al. 2009) but there is still room for needed improvement.

\section{Main Probe: Clustering features in redshift and real space}

Redshift surveys of galaxies provide us with one of the most powerful probes of the cosmological model via the study of galaxy clustering and redshift-space distortions. The shape of the power spectrum $P(k)$ of the galaxy distribution (on linear scales) is directly sensitive to the mean matter density $\Omega_{M}$ and its main constituents, such as the baryon density $\Omega_{b}$ and possi ble contributions from massive neutrinos. $\Omega_{b}$ is linked to specific regular features in $P(k)$, the so-called Baryonic Acoustic Oscillations (BAO), which connect the matter power spectrum to the equivalent oscillations in the $\mathrm{CMB}$ angular power spectrum. BAOs have in fact been detected in the $P(k)$ of the $2 \mathrm{dF}-$ GRS and SDSS surveys (e.g. Eisenstein et al. 2005). BAOs pro- $\Omega$ vide a "standard comoving rod" of $\sim 110 \mathrm{Mpc}$ that can be used to probe the expansion history of the Universe, $H(z)$. In addition to allowing us to reconstruct the 3D spatial distribution of galaxies, redshift surveys carry also important information on the growth rate of structures.

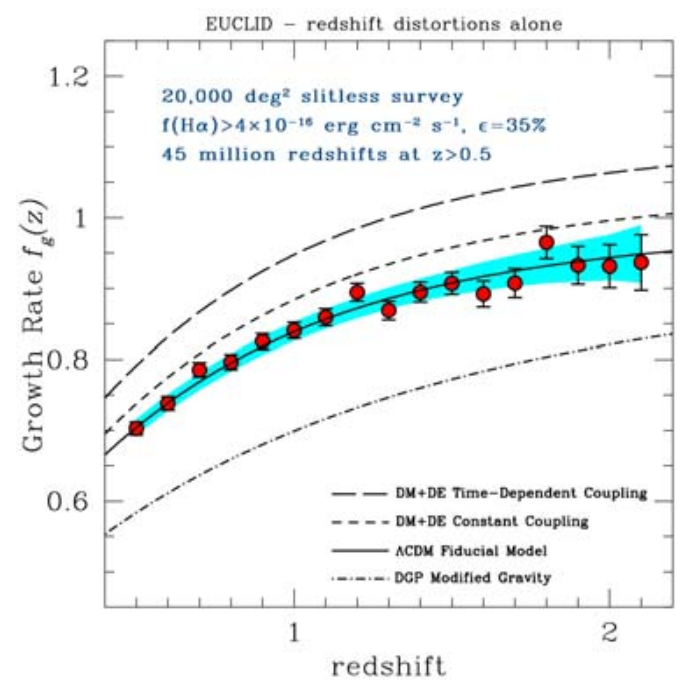

Fig. 6: Evolution of the growth rate of matter density perturbations: points are from simulations of the spectroscopic survey. $\Lambda \mathrm{CDM}$, coupled dark matter/dark energy models and DGP predictions are shown.

In fact, the measured redshift includes not only the cosmological expansion term, but also the Doppler contribution due to each galaxy's peculiar velocity. In the linear regime, this motion simply reflects the growth of structure and thus the actual 
value of the growth rate $f(z)$ (Kaiser 1987). This dynamical contribution introduces a measurable radial distortion in the reconstructed galaxy maps from redshift surveys, when redshifts are used as measures of distance. By measuring galaxy twopoint correlations along and perpendicular to the line-of-sight, one can quantify the distortions in terms of the linear compression parameter beta, which is related to the growth rate as $f(z)=\beta \times b$. In this expression, $b$ is the linear bias that depends on the kind of galaxies being used. It has recently been shown using the VVDS-Wide survey (Guzzo et al. 2008) that this method can be used as a test of dark energy, mapping $f(z)$ at different redshifts.

This provides an additional way to detect evidence for modified gravity as an alternative solution to the accelerated expansion problem. Conversely, if GR is assumed, this gives one further method to constrain the evolution of the equation of state $w(z)$, through a precise measurement of $\Omega_{M}(z)$ at different redshifts (Wang 2008).

\section{Complementary Cosmological Probes}

To better constrain and understand the present acceleration of the expansion there is a crucial need for multiple and complementary observational probes. One of them is the Integrated Sachs-Wolfe (ISW) effect (Sachs \& Wolfe 1967), arising from the time-variation of the scalar metric perturbations, imprinted in the CMB and it is of interest its correlation with the distribution of matter at lower redshifts (through the galaxy surveys). The ISW effect is sensitive to the low $\mathrm{z}$ decaying gravitational potential of the LSS and therefore is sensitive to the amount, equation of state and clustering properties of the dark energy. It is possible to detect the ISW effect through the crosscorrelation of the CMB with tracers of the LSS distribution (Rassat et al. 2007, Giannantonio et al. 2008). An optimal ISW survey has a minimum number density of sources of around 10 galaxy per $\operatorname{amin}^{2}$, covers a minimum sky fraction of the order of 0.5 and is reasonably deep, with a minimum median redshift of about 0.8 (Douspis et al., 2008): all requirements are well fulfilled by Euclid wide survey.

Galaxy clusters are the end products of the cosmic hierarchical build-up of small clumps into increasingly larger non linear structures up to $10^{15} M_{\odot}$. Since clusters arise from rare high peaks of primordial density perturbations, their number densities and mass distribution, i.e. their mass function $n(M, z)$, is highly sensitive to the matter density parameter and dark energy parameters which control the rate at which structure grows, as well as to the normalization of the matter power spectrum, $\sigma_{8}$, or to modified gravity (see e.g. Fig. 7, EYB).

A variety of method is used to select cluster samples. There will be samples already known by detections expected from other bands all-sky experiments such as eROSITA and Planck, but Euclid will improve in numbers, volumes and mass ranges. With Euclid one can target galaxy overdensities, either in projection only or by combining with redshift space information, with methods like the brightest cluster galaxy (maxBCG, Koester et al. 2007) or the optical richness by selecting galaxies along the red sequence. In addition, both weak (Bergé et al. 2007 ) and strong lensing can be used (expect $\approx 5000$ clusters

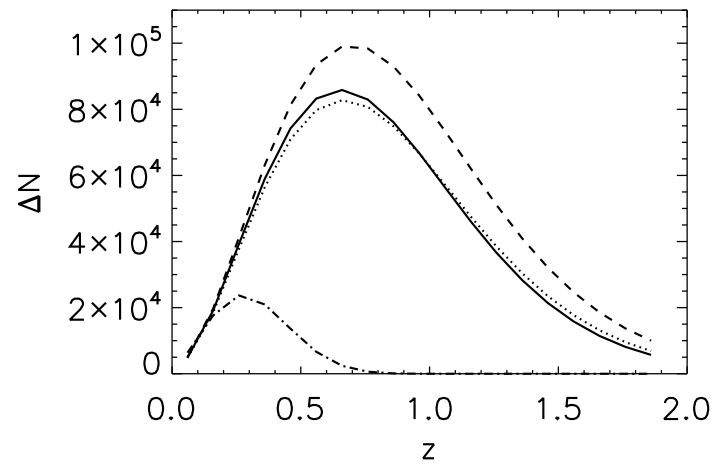

Fig. 7: Distribution of galaxy clusters in redshift bins of width $\Delta z=$ 0.1 for different cosmologies observed on $20,000 \mathrm{deg}^{2}$. All clusters above a mass limit of $5 \cdot 10^{13} h^{-1} M_{\odot}$ are selected. Lines show a $\Lambda \mathrm{CDM}$ model (solid), a $w=0.9$ model (dotted) and a modified gravity model $(\gamma=0.68$; dashed). The dot-dashed line is for a $\Lambda$ CDM model with the mass limit of the weak lensing $3-\sigma$ detection limit

with strong arcs). Strong lensing will help also in the selection of low mass systems (Limousin et al. 2009). It is important to stress that both lensing and redshifts (via virial estimates expect $\sim 500$ clusters with $N>20$ measured members- or caustics in redshift space, Diaferio 1999) will give improved mass estimates, density profiles, and in turn tighten the M- $\mathrm{L}_{X}$ relations (Rozo et al. 2009).

Supernovæ are observable in the repeated observations of the deep survey (NIR AB mags of 26 reached in $\approx 40$ visists each of depth 24, slitless spectroscopy with visits of $H \sim 19.5$ to a final $H \sim 21.5$ ). From these one can expect 1000- 2000 SNeIa to $z<0.7$ plus a further 1000-2000 to $z<1$ (detections only). NIR selection lessens the missed fraction in optical searches (CC SNe: $30 \%$ at $z=1,60 \%$ at $z=2$; SN Ia: $15 \%$ at $z=1$ and $35 \%$ at $z=2$, Mannucci et al. 2007). Other benefits are better control of dust extinction and a smaller scatter in Hubble diagram at longer rest-frame wavelengths. Euclid SNeIa will provide for cosmology much improved extinction corrections and rest-frame I-band Hubble diagram with thousands of objects to $z \sim 0.7$. For astrophysics will give accurate $\mathrm{SN}$ rates, including extincted $\mathrm{SNe}$, useful to constrain progenitor models, models of feedback processes, $\mathrm{SFH}$, and a better understanding of SNeIa physics for cosmology.

\section{Additional Topics and Legacy}

Here we can simply list some among several topics and stress the enormous legacy value, which can only be glimpsed at in terms of equivalents of the fundamental Sloan Digital Sky Survey [SDSS]. It is important to see Euclid also in perspective as the future main database from which extract targets for narrow field facilities, such as ALMA, JWST, ELT.

Imaging $(\mathrm{EYB})$ : one expects $\sim 2 \cdot 10^{9}$ galaxies with morpholgy and photoz, hence a factor $\sim \times 10^{3}$ the SDSS. From these one will get optical morphology and photoz for hundred millions of sources in other bands (SKA included). Of local interest: free-floating super-Jupiters in Young star-clusters, Milky 
Way satellites (faintest-end of dwarf galaxy luminosity function; radial distribution). Of interest for galaxies: rest-frame optical luminosities and stellar masses for the bulk of the galaxy population out to $\mathrm{z} 3$ (down to $0.1 \mathrm{M}_{*}$ at $z=1$ ), bulk of the unobscured star formation out to $z=6$ through rest $U V$, influence of the environment on galaxy structural and photometric evolution $\left(\sim 4 \cdot 10^{4}\right.$ lensing detected galaxy clusters at $\left.0.3<z<0.7\right)$. The rare but fundamental most luminous galaxies and AGNs at $z \sim 7-10$, high $z>2$ NIR detected clusters. Can also get constraints on the Luminosity Function at $z>6.5$ from the deep survey. The latter will be an unique bridge between JWST and ground based surveys $\left(\sim 10^{3} \times\right.$ Area of UltraVista in $0.1 \times$ time $)$. One also expects $\sim 10^{3}$ QSOs and $\sim 10^{5}$ galaxies to be lensed by galaxies. Interesting results on low mass planets are possible with a dedicated microlensing survey towards the galactic bulge and, similarly, a Galactic Plane survey in an extended mission would be the reference dataset for star formation and Galactic structure studies (EICSB).

Spectroscopy (EYB): a factor $\sim \times 70$ the SDSS. For galaxies (expect $\sim 10^{8}$ ): evolution of the multivariate distribution functions of star-forming galaxies, spectral estimates of extinction for higher fluxes with $H_{\beta}$ for $z>1.05$; the cosmic evolution of the star formation density and activity for $0.5<z<2$, and its relation to the LSS (expect $\sim 1.4 \cdot 10^{5}$ groups with $N>5$ members, and $\sim 500$ clusters with $N>20$ members); the evolution of the merger rate. Of interest for AGNs (expect $N \sim 10^{6}$ ): co-evolution with galaxy population, the fraction of AGN as a function of properties of the host galaxy; an unique sample of type $2 \mathrm{AGN}$ at $z>1$. One also has blind searches of high z QSO and extended $L y_{\alpha}$ emitters at $z>7$. Of interest for galactic science: ultra-cool dwarfs (L, T, Y) in the solar neighborhood and in star forming regions; results on the IMF at very low mass; the possibility of charting the unkown territory between the coolest dwarfs (T-type, $T \sim 1100 K$ ) and objects Jupiter-like $(T \sim 150 K)$.

\section{Conclusions}

Once realized, Euclid not only will provide us with quite precise and discriminating figures needed to better understanding the global picture, the main components and gravity characteristics, but will also yield enormous amount of priceless information to study e.g. at global, statistical level, the physical evolution of galaxies, clusters and large scale structures.

It is then worth stressing not only the areas of possible direct cross checks with SKA (i.e. large volumes weak lensing and BAO with different systematics) but also the wonderful complementarities: by joining the different information we will have finally available the information on both the arena (cosmology, laws of gravitational growth) and on the detailed behaviour of two main interacting players at low z, Dark Matter and Baryons. And for the latters we will able for hundred(s) million(s) galaxies to at last follow the whole non linear baryonic fate by going from the pristine fuel (HI content, a crucial information today missing but locally), via the flame $\left(H_{\alpha}\right)$, to the ashes (old stars and population fitting from NIR photometry).
Acknowledgements. The material summarized here is due to the efforts of hundreds of scientists who have been working in the ESA Euclid Science Study Team and in the EIC and ENIS consortia.

Partial support was given by the Italian Space Agency through the ASI contract "Euclid-Dune" number I/064/08/0

\section{References}

Amara, A. \& Réfregiér, A., 2008, MNRAS, 391, 228

Albrecht, A., Bernstein, G., Cahn, R., et al. 2006, ArXiv astroph/0609591 (DETF Report)

Amendola, L., Kunz, M., \& Sapone, D., 2008, Journal of Cosmology and Astro-Particle Physics, 4, 13

Bergé, J., et al., 2008, MNRAS, 385, 695.

Bordoloi, R., et al., 2009, ArXiv preprints

Bridle, S., et al., 2009, submitted to MNRAS, arXiv0908.0945

Chevallier, M. \& Polarski, D. 2001, International Journal of

Modern Physics D, 10, 213

Diaferio, A. 1999, MNRAS, 309, 610

Douspis, M., et al., 2008, A\&A, 485, 395.

Dvali, G., Gabadadze, G., \& Porrati, M. 2000, Physics Letters B, 485, 208 [DGP]

Eisenstein, et al. 2005, ApJ, 633, 560

Giannantonio, T., et al., 2008, Phys. Rev. D, 77(12), 123520

Guzzo L. et al. 2008, Nature, 451, 541

eavens, A. F., et al., 2006, MNRAS, 373, 105

Kaiser, N. 1987, MNRAS, 227, 1

Koester, B.P., et al. 2007a, ApJ, 660, 239

Komatsu, E., et al. 2009, ApJS, 180, 330

Laureijs R. et al. (Euclid Science Study Team), 2009, Euclid Assessment Report. ESA/SRE(2009)2 (Euclid "Yellow

Book" - [EYB]). http://sci.esa.int/sciencee/www/

object/index. cfm?fobjectid $=44880$

Limousin, M., et al., 2009, A\&A 502, 445

Linder, E. V., 2003, Phys. Rev. D, 68, 083504

Linder, E. V., 2005, Phys. Rev. D, 72, 043529

Mannucci, F., et al., 2007, MNRAS, 377, 1229

Massey, R., et al., 2007, Nature, 445, 286

Rassat, A., et al., 2007, MNRAS, 377, 1085

Refregier, A., 2003, ARAA, 41, 645

Réfrégier et al. 2009, Euclid Imaging Consortium Science Book[EICSB], ArXiv preprints

Rozo, E., et al. 2009, ApJ, 703, 601

Sachs, R. K. \& Wolfe, A. M., 1967, Astrophys. J., 147, 73

Wang, Y. 2008, Phys. Rev. D, 78, 123532

Wang, L. \& Steinhardt, P.J. 1998, ApJ, 508, 483 